\title{
The impact of repetitive navigated transcranial magnetic stimulation coil positioning and stimulation parameters on human language function
}

Nico Sollmann ${ }^{1,2}$, Sebastian $\| e^{1,2}$, Thomas Obermueller ${ }^{1,2}$, Chiara Negwer ${ }^{1,2}$, Florian Ringel $^{1,2}$, Bernhard Meyer ${ }^{1}$ and Sandro M Krieg ${ }^{1,2^{*}}$

\begin{abstract}
Background: Repetitive navigated transcranial magnetic stimulation (rTMS) in combination with object naming is able to elicit naming errors by stimulating language-related brain regions. However, stimulation results mainly depend on coil positioning and stimulation parameters, which have not been investigated since the implementation of neuronavigation to transcranial magnetic stimulation. Therefore, the following three parameters were systematically examined in the present study: coil angulation, stimulation frequency, and stimulation intensity.
\end{abstract}

Methods: Five healthy, right-handed subjects underwent rTMS language mapping of Broca's as well as Wernicke's areas of the left hemisphere. During mapping sessions, coil angulation was changed clockwise in $45^{\circ}$ steps, and the stimulation frequency and intensity were varied within a considerably wide range. For angulation, the anterior-posterior (ap) coil orientation was used as reference position.

Results: An angulation of $90^{\circ}$ to ap coil orientation led to the highest rate of naming errors within Broca's area, whereas an inhomogeneous distribution of angulations was observed during stimulation of Wernicke's area. Therefore, ap coil orientation, which is regarded as standard in rTMS language mapping, could not be approved as the optimal position. With regard to stimulation parameters, $20 \mathrm{~Hz}$ and 120\% of the resting motor threshold (RMT) were defined as optimal.

Conclusions: Coil angulation, stimulation frequency, and stimulation intensity have significant impacts on language impairment during rTMS mapping. The variation of only one of these parameters already leads to a clearer disruption of language performance. Therefore, individually adapted stimulation protocols have to be determined prior to language mapping in order to improve mapping results.

Keywords: Transcranial magnetic stimulation, Navigated brain stimulation, Cortical mapping, Language, Stimulation protocol, Object naming

\section{Background}

Over the last years, transcranial magnetic stimulation (TMS) has been increasingly used for diagnostic and therapeutic purposes. In general, TMS is a non-invasive method that induces an electrical field, which indirectly and transiently excites or inhibits pyramidal neurons.

\footnotetext{
* Correspondence: Sandro.Krieg@|rz.tum.de

'Department of Neurosurgery, Klinikum rechts der Isar, Technische Universität München, Ismaninger Str. 22, 81675 Munich, Germany ${ }^{2}$ TUM-Neuroimaging Center, Klinikum rechts der Isar, Technische Universität München, Ismaninger Str. 22, 81675 Munich, Germany
}

Navigated transcranial magnetic stimulation (nTMS), which is a combination of a TMS unit and a neuronavigation system, features a simultaneous 3D tracking and visualization of cortical stimulation points [1]. With regard to its therapeutic options, it has already been used for the treatment of a variety of psychiatric and neurological disorders, especially depression [2-4], chronic tinnitus [5-7], and chronic pain [8-10]. Concerning diagnostic purposes, nTMS has started to play an important role for neurosurgical operation planning as it can also be used to map functionally relevant brain

\section{Ciomed Central}

(c) 2015 Sollmann et al.; licensee BioMed Central. This is an Open Access article distributed under the terms of the Creative Commons Attribution License (http://creativecommons.org/licenses/by/4.0), which permits unrestricted use, distribution, and reproduction in any medium, provided the original work is properly credited. The Creative Commons Public Domain Dedication waiver (http://creativecommons.org/publicdomain/zero/1.0/) applies to the data made available in this article, unless otherwise stated. 
areas, which has surgical implications for resectable/nonresectable decision-making. Nowadays, as part of a multimodal setup, nTMS is primarily used for the preoperative mapping of motor- and language-related brain regions [11-16]. While established stimulation protocols are already available for nTMS motor mapping, there is no reliable and standardized stimulation protocol for the mapping of language-related brain areas by repetitive nTMS (rTMS). In general, rTMS language mapping results depend on a variety of different parameters, especially coil angulation, stimulation frequency, and stimulation intensity. Variation of only one of these parameters can already lead to a different TMS impact on language performance, which has been known since the publication of Epstein et al.'s examination of different stimulation settings [17]. Although it is one of the main approaches focused on the relationship between rTMS parameters and language impairment, this study does not provide a systematic examination of numerous coil angulations. However, the targeting of the stimulation coil is already known to be crucial, and small rotations can already alter rTMS language mapping results [18].

As further development and standardization of the rTMS language mapping procedure seems to be essential for the successful use of TMS technique, we systematically examined the effects of the three already mentioned parameters on language performance. Therefore, five healthy and purely right-handed subjects underwent language mapping of Broca's and Wernicke's areas of the left hemisphere through rTMS combined with an object naming task. Coil angulation, stimulation frequency, and stimulation intensity were varied, and the results of language disruption were evaluated.

\section{Methods \\ Subjects}

Five healthy subjects underwent rTMS language mapping of Broca's and Wernicke's areas of the left hemisphere. All volunteers indicated German as their mother tongue, and right-handedness was approved by the Edinburgh Handedness Inventory (EHI) in all subjects. Three volunteers were female, and two were male.

This study was conducted with the consent of the local ethics committee of Technische Universität München (registration number: 2793/10) and in accordance with the Declaration of Helsinki. Written informed consent was obtained from all volunteers prior to MR imaging.

\section{MRI data acquisition}

Before rTMS language mapping, all subjects underwent magnetic resonance imaging (MRI). This was performed on a 3-T MRI scanner combined with an eight-channel phased array head coil (Achieva 3 T, Philips Medical Systems, Best, The Netherlands B.V.) without intravenous contrast administration. The protocol for MRI data acquisition was repeatedly used and described in other rTMS language mapping studies [16,19-21]. The 3D MRI dataset was transferred to the nTMS system using the DICOM standard.

\section{rTMS language mapping Experimental setup}

rTMS language mapping was performed with the same Nexstim eXimia NBS system, version 4.3, with a NexSpeech $^{\bullet}$ module (Nexstim Oy, Helsinki, Finland) in all cases. In short, this system provides an electromagnetic stimulation coil in combination with a neuronavigation unit, which allows simultaneous 3D tracking of the coil and visualization of all stimulation sites [1]. During rTMS, the coil induces an electrical field, which is visualized over the individual 3D MRI reconstruction images of each volunteer's brain, and the intracranial stimulation points are saved for later examination $[1,22]$. Our setup follows the protocol of previous studies on rTMS language mapping [12,16,18-21,23-25]. Only biphasic stimulation pulses were applied throughout the mappings.

\section{Determination of the resting motor threshold}

As a part of preparation for rTMS language mapping, all volunteers underwent the same procedure to determine the individual resting motor threshold (RMT). Therefore, motor evoked potentials (MEP) of the right abductor pollicis brevis (APB) muscle were measured by an electromyography (EMG) unit while stimulation impulses were applied over the left-hemispheric motor cortex as described in earlier reports [13,14,26]. The induced electrical field was oriented perpendicular to the left-hemispheric precentral gyrus that was stimulated for RMT determination $[13,14,26]$.

\section{Object naming and baseline testing}

As a common and frequently used task for language function testing, object naming, which engages all three major language production functions (articulation, meaning, and form), was used for baseline testing and rTMS language mapping, as recently published [19-21,27].

During baseline testing, 131 colored photographs of familiar living and non-living objects were displayed on a screen in front of the volunteer at an inter-picture interval (IPI) of $2.5 \mathrm{~s}$ and without simultaneous stimulation. Every subject was instructed to name all objects in German as precisely and quickly as possible. Misnamed objects were immediately discarded from the object sequence. After the first baseline testing session, a second one with the stack of remaining images was performed in an analog way. The remaining objects, after second baseline testing, were used during the stimulation session. For later analysis, the baseline performances were digitally video recorded $[19,24]$. 


\section{Language mapping procedure}

After determination of the individual RMT and baseline testing, language mapping was performed in order to examine the impact of three different parameters on language performance: coil angulation, stimulation frequency, and stimulation intensity. Therefore, all volunteers underwent rTMS of Broca's and Wernicke's areas of the left hemisphere based on the following protocol:

1. A train of rTMS bursts was administered to Broca's and Wernicke's areas of the left hemisphere in order to identify one cortical site with clear and reproducible no-response errors each, based on the volunteer's and examiner's impressions and supported by video analysis. For determination of optimal cortical spots, mapping was performed with anterior-posterior (ap) coil orientation, $5 \mathrm{~Hz} / 5$ pulses (duration: $1.0 \mathrm{~s}$ ), and 100\% of the individual RMT.

These values are most frequently used as starting parameters in rTMS-based language investigations [19,21,28-30].

2. At these two sites with reproducible language impairments, coil angulation and stimulation frequency and intensity were varied according to the following chronological order:

a. Coil angulation (Figure 1): variation in steps of $45^{\circ}$ (beginning with ap coil orientation); 10 stimulations per position ( 8 positions $=80$ stimulations) with $5 \mathrm{~Hz} / 5$ pulses (duration: $1.0 \mathrm{~s}$ ) and $100 \%$ RMT.

b. Stimulation frequency: stimulation with $5,7,10$, and $20 \mathrm{~Hz} / 5$ pulses in ap and optimal coil orientation (as found in 2a); 10 stimulations per position and frequency ( 4 frequencies $\times 2$ positions $\times 10=80$ stimulations) with $100 \%$ RMT.

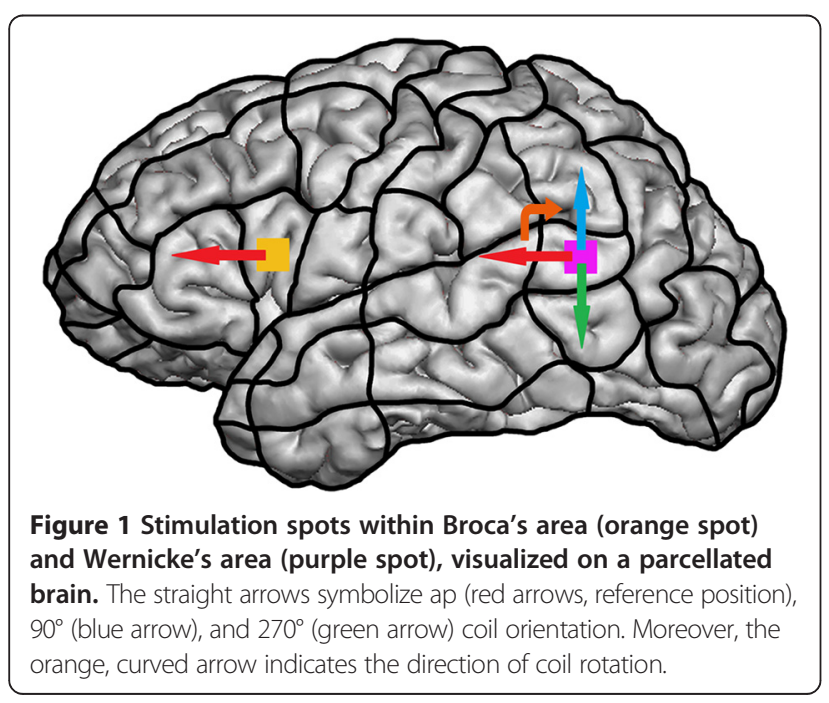

c. Stimulation intensity (\% RMT): stimulation with $100 \%, 80 \%$, and $120 \%$ RMT in ap (with $5 \mathrm{~Hz} / 5$ pulses) and optimal coil orientation (with the optimal frequency as found in $2 \mathrm{~b}$ ); 10 stimulations per position and intensity ( 3 intensities $\times 2$ positions $\times 10=60$ stimulations).

Broca's and Wernicke's areas were anatomically identified on each volunteer's MRI, meaning that during step 1 of our stimulation protocol, rTMS bursts were administered to the triangular and opercular parts of the inferior frontal gyrus (Broca's area) and to the posterior superior temporal gyrus and angular gyrus (Wernicke's area) to detect cortical sites with clear and reproducible no-response errors, as described above. Basically, both areas have shown to be especially prone to naming errors during rTMS in recent trials [24,25,31], which made them appropriate for systematic assessment of the three aforementioned parameters.

According to the stimulation protocol, a total number of $2 \times(80+80+60)=440$ stimulation trains were applied to each volunteer's cortex. The set of objects named correctly during baseline testing was displayed time-locked to a train of rTMS pulses. All objects were presented in a randomized endless loop mode with an IPI of $2.5 \mathrm{~s}$, and rTMS pulse trains were started $0 \mathrm{~ms}$ after the picture presentation onset. The coil was placed tangential to the skull in order to achieve maximum field induction $[17,24,32]$. For investigating the impact of coil orientation on language function, the angulation of the magnetic coil was changed during the IPI after 10 stimulations. We defined the ap orientation as the starting position, which means that the corresponding electrical field generated by the magnetic coil is angulated horizontally with respect to a line between external acoustic meatus and nasion (Figure 1). This position is commonly regarded as the standard position for rTMS-based language mappings $[17,19,24,33]$.

By causing a virtual functional lesion, rTMS is able to identify cortical regions causally related to language functions [34-36]. For an objective and detailed analysis, all individual mapping session performances were digitally video recorded $[19,24]$.

To evaluate discomfort during stimulation for safety and interpretability of the disrupted naming performance, each volunteer was asked to rate perceived pain according to the visual analog scale (VAS) from 0 (no pain) to 10 (maximum pain) divided into temporal muscle pain and pain during rTMS at convexity.

\section{Data analysis}

All language mapping data were examined based on the videos after the investigation as described previously $[16,19-21,24]$. Any rTMS-induced disturbance of language 
was compared with the corresponding baseline performance, and clear naming errors were counted [37].

Moreover, optimal mapping parameters and optimal coil angulation were defined as the settings that lead to the highest number of language disturbances in total. Coil angulation was measured with ap coil orientation or gyrus as references (Figure 1). For these optimal settings, electrical field strength was evaluated at Broca's and Wernicke's area stimulation sites.

A chi-square test or Mann-Whitney-Wilcoxon test was performed to test the distribution of attributes. Results are presented as mean \pm standard deviation (SD) or individual scores. For interpretation, $P$ values were calculated, and $P<0.05$ was considered significant.

\section{Results}

\section{Subject-related characteristics}

Relevant subject-related characteristics, including age, handedness score based on the EHI, number of correctly named baseline objects, individual RMT, and pain score, according to the VAS are provided in Table 1.

\section{rTMS language mapping}

Ap coil orientation was defined as the optimal position in no case (Tables 2 and 3, Figures 2a,b and 3a,b). Higher stimulation frequencies and higher stimulation intensities led to an increased number of naming errors with regard to Broca's and Wernicke's areas in general (Tables 2, 4, and 5). In this context, within Broca's area, an angulation of $90^{\circ}$ to ap coil orientation led to the highest number of naming errors in three out of five cases (Tables 2 and 3, Figure 2a). Concerning coil orientation in relation to the reference gyrus, $0^{\circ}$ was defined as optimal in three cases during rTMS language mapping of Broca's area (Tables 2 and 3, Figure 2b). For Wernicke's area, an inhomogeneous distribution of coil angulations relative to ap coil orientation and to the reference gyrus was observed (Tables 2 and 3, Figure 3a,b). Furthermore, $20 \mathrm{~Hz}$ was defined as the optimal frequency in two out of five cases within Broca's area and in three out of five cases within Wernicke's area (Tables 2 and 4). During stimulation of Broca's and Wernicke's areas, an intensity of $120 \%$ RMT was defined as optimal in three out of five cases, whereas
$100 \%$ RMT led to the most language disturbances in two out of five cases (Tables 2 and 5).

\section{Discussion}

\section{Coil angulation}

The most interesting finding seems to be the fact that ap coil orientation, which is regarded as the standard position in rTMS language mapping $[17,19,24,33]$, was defined as the optimal position in no case (Tables 2 and 3, Figures 2a,b and 3a,b). With regard to Broca's area, Epstein et al., who tried to define optimal and tolerable TMS parameters for safe cortical language mapping in a cohort of six individuals, came to the conclusion that strict horizontal alignment of the induced electrical field elicits the highest rate of no-response errors [17]. In their study, they performed TMS language mapping with coil angulations of $0^{\circ}$ and $90^{\circ}$. Despite the fact that the authors only used two different angulations (rather than a larger range of orientations, as we did in our study), it is obvious that our results are not in concordance with their results at all. They also described pain as clearly more intensive during stimulation with $90^{\circ}$ due to the potential direct stimulation of temporal muscle fibers [17] - an observation that cannot be approved by our data. Although our study results do not include explicit VAS scores for perceived pain during stimulation at each coil orientation, each subject was asked to rate the pain immediately after stimulation. None of the five volunteers enrolled in our investigation described one special angulation as more painful than another. rTMS of Broca's area is not completely painless under any circumstances, but with regard to coil positioning, none of the eight angulations led to more or less discomfort compared to the others.

Up to now, the literature about the exact correlation between coil orientation in relation to the reference gyrus or ap direction and error evocation has been very rare and has never been systematically investigated. As has been mentioned, some authors have reported that variations of coil angulation led to different rTMS language mapping results in their studies $[17,18]$, but an explanation of this observation is missing. Therefore, a reasonable, literature-based analysis of our results

Table 1 Subject-related characteristics

\begin{tabular}{|c|c|c|c|c|c|c|c|c|}
\hline \multicolumn{2}{|l|}{ Subject } & $\# 1$ & $\# 2$ & $\# 3$ & $\# 4$ & $\# 5$ & Mean \pm SD & $P$ values \\
\hline \multicolumn{2}{|l|}{ Age (years) } & 24 & 26 & 29 & 26 & 23 & $25.6 \pm 2.3$ & - \\
\hline \multicolumn{2}{|c|}{ Handedness (EHI) } & 100 & 57 & 100 & 100 & 60 & $83.4 \pm 22.8$ & \\
\hline \multicolumn{2}{|c|}{ Correctly named baseline objects (out of 131) } & 111 & 115 & 121 & 113 & 105 & $113.0 \pm 5.8$ & \\
\hline \multicolumn{2}{|c|}{ RMT (\% output) } & 40 & 28 & 42 & 41 & 35 & $37.2 \pm 5.8$ & \\
\hline \multirow[t]{2}{*}{ Pain (VAS) } & Broca & 7 & 5 & 2 & 4 & 5 & $4.6 \pm 1.8$ & $<0.0001$ \\
\hline & Wernicke & 2 & 1 & 1 & 2 & 3 & $1.8 \pm 0.8$ & \\
\hline
\end{tabular}


Table 2 Summary of stimulation parameters and coil angulations

\begin{tabular}{|c|c|c|c|c|c|c|c|c|c|c|c|c|}
\hline \multirow{2}{*}{ Subject } & & \multicolumn{5}{|c|}{ Broca } & \multicolumn{5}{|c|}{ Wernicke } & \multirow{2}{*}{$\begin{array}{l}P \text { values } \\
-\end{array}$} \\
\hline & & $\# 1$ & \#2 & \#3 & \#4 & \#5 & $\# 1$ & \#2 & \#3 & \#4 & \#5 & \\
\hline \multirow[t]{2}{*}{ Optimal coil angulation (in ${ }^{\circ}$ ) } & To ap orientation & 225 & 90 & 90 & 135 & 90 & 90 & 45 & 180 & 270 & 135 & 0.8288 \\
\hline & To reference gyrus & 135 & 0 & 0 & 45 & 0 & 0 & 315 & 90 & 180 & 45 & 0.1945 \\
\hline \multicolumn{2}{|c|}{ Optimal stimulation frequency (in $\mathrm{Hz}$ ) } & 10 & 10 & 20 & 20 & 7 & 20 & 20 & 20 & 7 & 10 & 0.7337 \\
\hline \multicolumn{2}{|c|}{ Optimal stimulation intensity (\% RMT) } & 100 & 120 & 120 & 120 & 100 & 120 & 100 & 100 & 120 & 120 & 0.9025 \\
\hline \multicolumn{2}{|l|}{ Electrical field strength (in V/m) } & 85 & 120 & 95 & 100 & 70 & 95 & 75 & 105 & 90 & 75 & 0.7526 \\
\hline
\end{tabular}

regarding coil orientation seems to be crucial. As one interpretation of our rTMS mapping results, it seems to be possible that different coil angulations interfere with different subcortical tracts, which can lead to an angulation-correlated grade of language impairment. In this context, angulation changes of an nTMS coil placed on the precentral gyrus for cortical motor mapping can lead to an increase in motor response latency out of the commonly known latency range of monosynaptic MEPs [38], meaning that nTMS presumably stimulated different and more distant motor-related structures in an angulation-dependent way. The same might principally be true for rTMS language mapping, meaning that different coil angulations could interfere with slightly different language-related tracts despite the spot of cortical stimulation stays the same. However, this hypothesis is not yet supported by the previous literature, and it remains questionable whether it can be justified in the light of future investigations.

Furthermore, $P$ values calculated for the comparison of Broca's $v s$. Wernicke's optimal coil angulations in relation to the reference gyrus and ap orientation indicate no significance (Table 2). This finding shows that, at least in our study, there is no correlation between the optimal coil orientations at these two areas for rTMS language mapping, which means that the coil angulation at one of these spots cannot predict the other one's optimal angulation. Therefore, coil angulations should be determined separately for Broca's and Wernicke's areas in order to improve the mapping results of the individual subject. However, the small sample size of this pilot study limits the result.

For rTMS language mapping of a single cortical spot, testing of different coil angulations in order to obtain optimal mapping results seems applicable. In contrast, a variation of coil orientation during stimulation of large areas or whole hemispheres is likely to become a demanding or even impossible task for the examiner, as he/she would have to change coil orientation and coil localization simultaneously within the IPI. Therefore, further investigations to detect single rTMS coil angulations that lead to optimal language impairment among most individuals are highly preferable. Because the cohort of our study is too small and optimal coil angulations for stimulation of Wernicke's area were distributed inhomogeneously among all volunteers, a definite recommendation for optimal coil orientation for rTMS language mapping in general cannot be made yet. Nonetheless, since previous studies on non-navigated rTMS language mapping did not investigate or discuss the matter of coil orientation at all, the results of this first systematic trial can be seen as the base for further studies on this topic $[17,24,33,39,40]$. To also evaluate the influence of subcortical or cortico-cortical fiber tracts, a new trial will have to combine rTMS language mapping with diffusion tensor imaging fiber tracking.

Table 3 Distribution of naming errors in relation to coil angulations

\begin{tabular}{|c|c|c|c|c|c|c|c|c|c|c|c|c|}
\hline \multirow[b]{2}{*}{ Subject } & \multicolumn{6}{|c|}{ Broca } & \multicolumn{6}{|c|}{ Wernicke } \\
\hline & $\# 1$ & $\# 2$ & $\# 3$ & $\# 4$ & $\# 5$ & Totals & $\# 1$ & $\# 2$ & $\# 3$ & $\# 4$ & $\# 5$ & Totals \\
\hline $0^{\circ}$ & 5 & 3 & 9 & 6 & 8 & 31 & 3 & 2 & 6 & 3 & 8 & 22 \\
\hline $45^{\circ}$ & 2 & 4 & 5 & 4 & 7 & 22 & 2 & 4 & 1 & 3 & 6 & 16 \\
\hline $90^{\circ}$ & 5 & 7 & 10 & 6 & 9 & 37 & 6 & 3 & 2 & 4 & 5 & 20 \\
\hline $135^{\circ}$ & 5 & 4 & 4 & 7 & 6 & 26 & 3 & 2 & 2 & 4 & 10 & 21 \\
\hline $180^{\circ}$ & 3 & 1 & 3 & 6 & 8 & 21 & 4 & 2 & 7 & 2 & 8 & 23 \\
\hline $225^{\circ}$ & 6 & 3 & 5 & 4 & 8 & 26 & 3 & 3 & 4 & 3 & 7 & 20 \\
\hline $270^{\circ}$ & 4 & 3 & 7 & 4 & 7 & 25 & 3 & 1 & 4 & 5 & 7 & 20 \\
\hline $315^{\circ}$ & 3 & 2 & 4 & 3 & 8 & 20 & 1 & 2 & 4 & 2 & 6 & 15 \\
\hline Optimal angulation (in ${ }^{\circ}$ ) & 225 & 90 & 90 & 135 & 90 & - & 90 & 45 & 180 & 270 & 135 & - \\
\hline
\end{tabular}




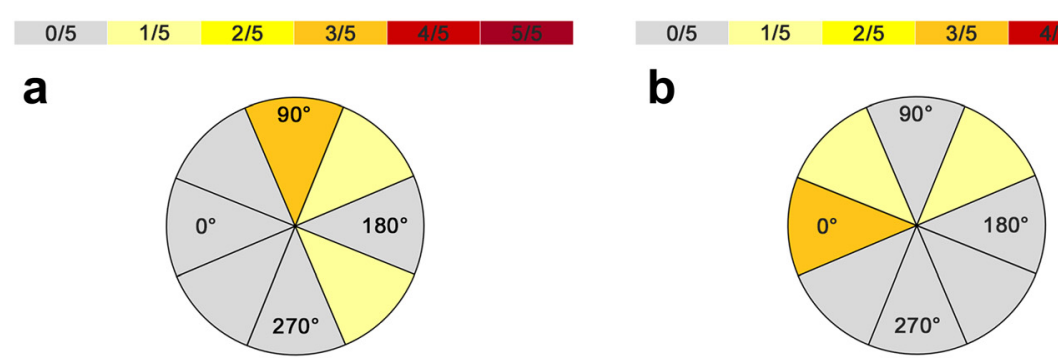

Figure 2 The circle diagram visualizes the distribution of optimal coil angulations within Broca's area in relation to ap coil orientation (a) as well as in relation to the reference gyrus (b), and the number of subjects who were prone to most of the errors at these angulations.

\section{Stimulation frequency}

In general, higher stimulation frequency was correlated with higher numbers of naming errors according to our stimulation results (Tables 2 and 4). When our stimulation frequency findings are compared to those of Epstein et al., results are not in concordance once again [17]. In their study, the authors used five different frequencies (2, 4, 8, 16, and $32 \mathrm{~Hz})$ for rTMS language mapping [17]. Their results illustrated that slower repetition rates of TMS pulses led to clearer language impairment with special regard to no-response errors because stimulation with 4 and $8 \mathrm{~Hz}$ elicited speech arrests in six out of six subjects, whereas rTMS with 16 and $32 \mathrm{~Hz}$ only lead to speech arrests in two out of six subjects [17]. In our study, the highest examined stimulation intensity $(20 \mathrm{~Hz})$ was correlated with most of the language disturbances that were observed, whereas low frequencies ( 5 and $7 \mathrm{~Hz}$ ), which were comparable to those used in the study of Epstein et al., only elicited clear naming errors occasionally [17]. In a different study of our research group, a study that used 5 and $7 \mathrm{~Hz}$ for triplicate language mapping among 10 healthy volunteers, stimulation with higher frequency $(7 \mathrm{~Hz})$ again elicited more language disturbances than low-frequency mapping [21]. Moreover, when not only focusing on one modality for the identification of human language-related brain areas, at electrocorticography (ECoG), for example, a relatively high frequency is usually considered optimum for language localization, and this can lead to the assumption that higher frequencies would be more effective for rTMS language mapping as well $[17,41]$.

In a study of Pascual-Leone et al., rTMS language mapping was performed with frequencies of 8,16 , and $25 \mathrm{~Hz}$ in six patients, and 8 and $16 \mathrm{~Hz}$ led to the greatest number of no-response errors in three patients each [39]. Therefore, this study seems to partially correspond with Epstein et al.'s study and our present study because half of the individuals were prone to naming errors during low frequency and half of the subjects were prone to naming errors during high-frequency rTMS $[17,39]$. In a study published this year, authors Rogic et al. performed language mapping with high-frequency rTMS $(12 \mathrm{~Hz})$ and found that language disruptions were successfully produced in all subjects [25]. Moreover, further literature review also tends to indicate that language impairment is more likely to be caused by high frequency than by low-frequency stimulation $[40,42]$.

In addition, some authors reported that rTMS using high frequency is related to a higher rate of unclear language disturbances due to dysarthria or intolerable pain $[17,39]$. We came to the same results regarding these two aspects after evaluation of our study in general. However, video-based error counting of high-frequency mapping sessions was not significantly more difficult than low-frequency examinations, and dysarthria was not a severe problem for data analysis among our study
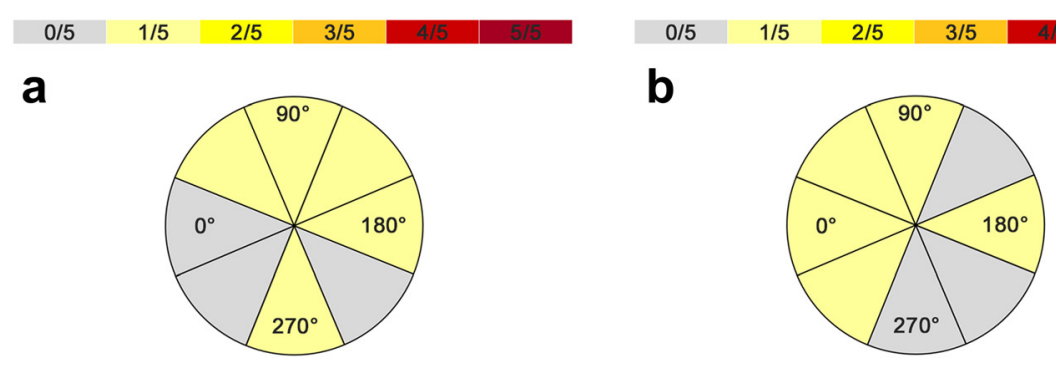

Figure 3 The circle diagram visualizes the distribution of optimal coil angulations within Wernicke's area in relation to ap coil orientation (a) as well as in relation to the reference gyrus (b), and the number of subjects who were prone to most of the errors at these angulations. 
Table 4 Distribution of naming errors in relation to stimulation frequencies

\begin{tabular}{|c|c|c|c|c|c|c|c|c|c|c|c|c|}
\hline \multirow[b]{2}{*}{ Subject } & \multicolumn{6}{|c|}{ Broca } & \multicolumn{6}{|c|}{ Wernicke } \\
\hline & $\# 1$ & \#2 & \#3 & $\# 4$ & \#5 & Totals & $\# 1$ & \#2 & \#3 & \#4 & \#5 & Totals \\
\hline $5 \mathrm{~Hz}$ ap & 2 & 3 & 6 & 3 & 9 & 23 & 3 & 3 & 3 & 2 & 8 & 19 \\
\hline $5 \mathrm{~Hz}$ opt. & 2 & 4 & 9 & 6 & 7 & 28 & 4 & 3 & 6 & 3 & 7 & 23 \\
\hline $7 \mathrm{~Hz}$ ap & 3 & 4 & 2 & 4 & 9 & 22 & 2 & 3 & 2 & 2 & 8 & 17 \\
\hline 7 Hz opt. & 4 & 3 & 7 & 6 & 10 & 30 & 4 & 1 & 5 & 6 & 8 & 24 \\
\hline $10 \mathrm{~Hz}$ ap & 2 & 3 & 9 & 3 & 10 & 27 & 3 & 4 & 6 & 4 & 9 & 26 \\
\hline 10 Hz opt. & 8 & 6 & 9 & 4 & 9 & 36 & 2 & 4 & 6 & 4 & 10 & 26 \\
\hline $20 \mathrm{~Hz}$ ap & 4 & 5 & 9 & 6 & 9 & 33 & 3 & 3 & 5 & 3 & 9 & 23 \\
\hline 20 Hz opt. & 4 & 5 & 10 & 7 & 9 & 35 & 5 & 5 & 8 & 5 & 9 & 32 \\
\hline Optimal frequency (in $\mathrm{Hz}$ ) & 10 & 10 & 20 & 20 & 7 & - & 20 & 20 & 20 & 7 & 10 & - \\
\hline
\end{tabular}

cohort. In addition, no volunteer requested a reduction of stimulation frequency due to intolerable discomfort or pain. Therefore, it is clear that rTMS with frequencies up to $20 \mathrm{~Hz}$, as used in this study, turned out to be safe and tolerable for the individual subject.

$P$ values comparing optimal stimulation frequencies for Broca's vs. Wernicke's rTMS language mapping indicate no significance (Table 2), and this shows that there is no specific correlation between the optimal frequencies of these two areas in our study. Therefore, at least in our study, the stimulation frequency determined for one of these spots is not likely to be able to predict the optimal frequency for the other one. This leads to the assumption that stimulation frequencies for rTMS language mapping should be determined separately for Broca's and Wernicke's areas.

In summation, as mentioned before, a higher stimulation frequency is correlated with a higher number of naming errors within Broca's area and also within Wernicke's area in our study. This finding indicates that high-frequency rTMS is needed to successfully disrupt linguistic functions, whereas low-frequency rTMS is not able to interfere with this on the same level [33]. Therefore, we come to the conclusion that languagerelated cortical areas are more prone to errors when stimulated with higher frequencies.

\section{Stimulation intensity}

In keeping with our findings concerning stimulation frequencies, higher stimulation intensity also led to higher numbers of language disturbances than lower intensities (Tables 2 and 5). Epstein et al., for example, started with a stimulation intensity of $120 \%$ RMT and increased it, if necessary, up to $150 \%$ RMT [17]. The ranges of their and our applied intensities have only 120\% RMT in common, which leads to the highest error number in our study. Epstein et al. did not systematically examine the single impact of stimulation intensities on human language function in their study, but it featured a positive correlation of intensity and discomfort [17], which is congruent with our findings. In Pascual-Leone et al.'s study, rTMS induced more reproducible naming errors when performed with high stimulation intensities [39]. Moreover, in Epstein et al., the authors described the occurrence of complete speech arrest, on average, at $116 \%$ RMT (range: $100 \%$ to $137 \%$ RMT) and stated that stronger magnetic stimulation is likely to produce more prominent effects on language functions [43], which are compatible with our stimulation results.

Similar to the $P$ values comparing optimal coil angulations and stimulation frequencies, $P$ values for the comparison of optimal intensities (Broca vs. Wernicke) indicate no statistically significant difference (Table 2). Therefore,

Table 5 Distribution of naming errors in relation to stimulation intensities

\begin{tabular}{|c|c|c|c|c|c|c|c|c|c|c|c|c|}
\hline \multirow[b]{2}{*}{ Subject } & \multicolumn{6}{|c|}{ Broca } & \multicolumn{6}{|c|}{ Wernicke } \\
\hline & $\# 1$ & $\# 2$ & $\# 3$ & $\# 4$ & $\# 5$ & Totals & $\# 1$ & $\# 2$ & $\# 3$ & $\# 4$ & \#5 & Totals \\
\hline 100\% RMT 5 Hz ap & 3 & 2 & 7 & 3 & 7 & 22 & 3 & 2 & 7 & 4 & 7 & 23 \\
\hline 120\% RMT $5 \mathrm{~Hz}$ ap & 4 & 3 & 10 & 5 & 7 & 29 & 3 & 3 & 7 & 5 & 10 & 28 \\
\hline 80\% RMT $5 \mathrm{~Hz}$ ap & 2 & 2 & 3 & 3 & 6 & 16 & 3 & 2 & 2 & 3 & 5 & 15 \\
\hline 100\% RMT opt. & 7 & 3 & 9 & 4 & 10 & 33 & 4 & 5 & 10 & 4 & 9 & 32 \\
\hline 120\% RMT opt. & 5 & 4 & 10 & 5 & 8 & 32 & 5 & 2 & 8 & 6 & 10 & 31 \\
\hline 80\% RMT opt. & 5 & 3 & 9 & 4 & 6 & 27 & 4 & 3 & 7 & 5 & 7 & 26 \\
\hline Optimal intensity (\% RMT) & 100 & 120 & 120 & 120 & 100 & - & 120 & 100 & 100 & 120 & 120 & - \\
\hline
\end{tabular}


we suggest that rTMS stimulation intensities should also be determined separately for Broca's and Wernicke's areas to optimize the impacts of rTMS on language-related brain areas. Mainly due to the relatively small size of the examined cohort, this recommendation has to be confirmed by future studies on rTMS language mapping.

As a consequence of our present study and literature review, the ranges for optimal stimulation intensities do not seem to be as controversial as those for coil angulations or for stimulation frequencies. Therefore, we can conclude that good rTMS language mapping results can already be achieved regularly with $100 \%$ RMT in most of individuals, but results can be improved significantly when higher intensities are used. This can be due to the fact that low stimulation intensities are able to interfere with language-related neuronal networks for only a short amount of time or to even enhance language functions, whereas high-intensity rTMS is likely to evoke a longer lasting effect on language functions, which results in an increased language impairment [33].

\section{Selection of stimulation targets}

Previous language mapping approaches and personal experience have shown that left-hemispheric Broca's area and Wernicke's area are especially prone to naming errors during rTMS [24,25,31], which qualified both regions as appropriate stimulation targets for assessing the impact of rTMS intensity, frequency, and coil angulation on language performance during an object naming paradigm. Although rTMS to these cortical regions has routinely led to comparatively high error rates when compared with other stimulation spots, we have to be aware of the fact that the impact of the parameters evaluated in the present study might potentially be different with respect to other areas. Therefore, further studies investigating more distributed language-related areas might be helpful.

Moreover, the terms 'Broca's area' and 'Wernicke's area' are used to describe the spatio-anatomical cortical areas that were stimulated in the present study. In this context, we have to be aware of the modern literature favoring a widespread network responsible for language production and comprehension, which includes, but clearly exceeds the regions of Broca's and Wernicke's areas [44,45]. As aforementioned, the selection of the study's stimulation targets is predominantly based on practical rTMS language mapping experience; therefore, any investigation of actual language organization models using rTMS is out of the scope of the present study.

\section{Safety aspects of rTMS}

Although no adverse events were observed in the present study, we have to be aware of the principal risk of undesirable effects in the course of rTMS [46-48]. Concerning possible side effects, Wassermann mainly reports on epileptic seizures but also on hearing loss or effects on mood and cognition, for example [48]. Indeed, early TMS-based studies reported on the induction of seizures within a minority of patients [49,50], but none of the TMS-induced seizures described in previous publications led to permanent physical sequelae. More important, recent rTMS language trials of our and other groups did not lead to any seizures or other adverse events (except temporary headache) in patients or healthy volunteers [19,21,23,28-30].

Although several rTMS language mapping studies were published over the last years, distinct evidencebased data concerning limitations of stimulation intensity, stimulation frequency, and the number of applied stimulation pulses are still lacking regarding language mapping in particular. However, due to the fact that rTMS-based language mapping is usually performed with $80 \%$ to $120 \%$ RMT and a frequency of $\leq 20 \mathrm{~Hz}$ $[19,21,23,28-30]$, the currently available safety limits should remain unaffected $[47,48]$.

\section{Conclusions}

Coil angulation, stimulation frequency, and stimulation intensity have a significant impact on language performance during rTMS language mapping. Variation of only one of these parameters already leads to a clearer impairment of language performance. Therefore, individually adapted stimulation parameters must be determined prior to language mapping in order to improve mapping results for each subject. Moreover, even high stimulation frequencies and intensities are safe and tolerable, at least in this small series.

\section{Abbreviations}

Ap: anterior-posterior; APB: abductor pollicis brevis muscle; DCS: direct cortical stimulation; ECoG: electrocorticography; EHI: Edinburgh Handedness Inventory; EMG: electromyography; Hz: hertz; IPI: inter-picture interval; MEP: motor evoked potentials; MRI: magnetic resonance imaging; TMS: transcranial magnetic stimulation; nTMS: navigated transcranial magnetic stimulation; RMT: resting motor threshold; rTMS: repetitive navigated transcranial magnetic stimulation; SD: standard deviation; VAS: visual analog scale.

\section{Competing interests}

The authors declare that they have no competing interests.

\section{Authors' contributions}

NS drafted the manuscript, was responsible for data acquisition, handled the acquired data, and performed literature research. SI, TO, and CN read and approved the final manuscript. FR and BM approved and corrected the final version of the manuscript. SK was responsible for the concept and design of the study and corrected the final version. All authors read and approved the final manuscript.

Authors' information

NS, SI, TO, CN, and SK are medical doctors. BM is chairman, and FR is vice chairman of the department. SK and FR are consultants for BrainLAb AG (Feldkirchen, Germany).

\section{Acknowledgements}

We thank the commission for clinical research of our university for funding SK within the scope of a faculty-intern grant. The study was financed by 
institutional grants from the Department of Neurosurgery and by funding through the commission for clinical research of our university.

\section{Received: 18 December 2014 Accepted: 19 March 2015 Published online: 01 April 2015}

\section{References}

1. Ruohonen J, Karhu J. Navigated transcranial magnetic stimulation. Neurophysiol Clin Clin Neurophysiol. 2010;40(1):7-17.

2. Fox MD, Buckner RL, White MP, Greicius MD, Pascual-Leone A. Efficacy of transcranial magnetic stimulation targets for depression is related to intrinsic functional connectivity with the subgenual cingulate. Biol Psychiatry. 2012;72(7):595-603.

3. Chen J, Zhou C, Wu B, Wang Y, Li Q, Wei Y, et al. Left versus right repetitive transcranial magnetic stimulation in treating major depression: a metaanalysis of randomised controlled trials. Psychiatry Res. 2013;210(3):1260-4.

4. Berlim MT, Van den Eynde F, Daskalakis ZJ. A systematic review and metaanalysis on the efficacy and acceptability of bilateral repetitive transcranial magnetic stimulation (rTMS) for treating major depression. Psychol Med. 2013;43(11):2245-54.

5. Folmer RL. Repetitive transcranial magnetic stimulation for tinnitus. Arch Otolaryngol Head Neck Surg. 2011;137(7):730. author reply 730-32.

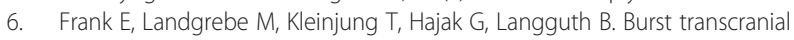
magnetic stimulation for the treatment of tinnitus. CNS Spectr. 2010;15(8):536-7.

7. Kim BG, Kim DY, Kim SK, Kim JM, Baek SH, Moon IS. Comparison of the outcomes of repetitive transcranial magnetic stimulation to the ipsilateral and contralateral auditory cortex in unilateral tinnitus. Electromagn Biol Med. 2014;33(3):211-5.

8. Fricova J, Klirova M, Masopust V, Novak T, Verebova K, Rokyta R. Repetitive transcranial magnetic stimulation in the treatment of chronic orofacial pain. Physiol Res. 2013;62 Suppl 1:S125-34.

9. Cheng JK. Repetitive transcranial magnetic stimulation - an alternative treatment for chronic refractory pain. Acta Anaesthesiol Taiwan. 2013;51(2):51-2.

10. Lefaucheur JP. The use of repetitive transcranial magnetic stimulation (rTMS) in chronic neuropathic pain. Neurophysiol Clin Clin Neurophysiol. 2006;36(3):117-24.

11. Takahashi S, Vajkoczy P, Picht T. Navigated transcranial magnetic stimulation for mapping the motor cortex in patients with rolandic brain tumors. Neurosurg Focus. 2013;34(4):E3.

12. Tarapore PE, Tate MC, Findlay AM, Honma SM, Mizuiri D, Berger MS, et al. Preoperative multimodal motor mapping: a comparison of magnetoencephalography imaging, navigated transcranial magnetic stimulation, and direct cortical stimulation. J Neurosurg. 2012;117(2):354-62.

13. Krieg SM, Shiban E, Buchmann N, Gempt J, Foerschler A, Meyer B, et al. Utility of presurgical navigated transcranial magnetic brain stimulation for the resection of tumors in eloquent motor areas. J Neurosurg. 2012;116(5):994-1001.

14. Krieg SM, Shiban E, Buchmann N, Meyer B, Ringel F. Presurgical navigated transcranial magnetic brain stimulation for recurrent gliomas in motor eloquent areas. Clin Neurophysiol. 2013;124(3):522-7.

15. Picht T, Schmidt S, Brandt S, Frey D, Hannula H, Neuvonen T, et al. Preoperative functional mapping for rolandic brain tumor surgery: comparison of navigated transcranial magnetic stimulation to direct cortical stimulation. Neurosurgery. 2011;69(3):581-8. discussion 588.

16. Sollmann N, Picht T, Makela JP, Meyer B, Ringel F, Krieg SM. Navigated transcranial magnetic stimulation for preoperative language mapping in a patient with a left frontoopercular glioblastoma. J Neurosurg. 2013;118(1):175-9.

17. Epstein CM, Lah JJ, Meador K, Weissman JD, Gaitan LE, Dihenia B. Optimum stimulus parameters for lateralized suppression of speech with magnetic brain stimulation. Neurology. 1996:47(6):1590-3.

18. Tarapore PE, Findlay AM, Honma SM, Mizuiri D, Houde JF, Berger MS, et al. Language mapping with navigated repetitive TMS: proof of technique and validation. Neuroimage. 2013;82:260-72.

19. Picht T, Krieg SM, Sollmann N, Rosler J, Niraula B, Neuvonen T, et al. A comparison of language mapping by preoperative navigated transcranial magnetic stimulation and direct cortical stimulation during awake surgery. Neurosurgery. 2013;72(5):808-19.
20. Krieg SM, Sollmann N, Hauck T, Ille S, Meyer B, Ringel F. Repeated mapping of cortical language sites by preoperative navigated transcranial magnetic stimulation compared to repeated intraoperative DCS mapping in awake craniotomy. BMC Neurosci. 2014;15:20.

21. Sollmann N, Hauck T, Hapfelmeier A, Meyer B, Ringel F, Krieg SM. Intra- and interobserver variability of language mapping by navigated transcranial magnetic brain stimulation. BMC Neurosci. 2013;14:150.

22. Ruohonen J, Ilmoniemi RJ. Modeling of the stimulating field generation in TMS. Electroencephalogr Clin Neurophysiol Suppl. 1999;51:30-40.

23. Rosler J, Niraula B, Strack V, Zdunczyk A, Schilt S, Savolainen P, et al. Language mapping in healthy volunteers and brain tumor patients with a novel navigated TMS system: evidence of tumor-induced plasticity. Clin Neurophysiol. 2014;125(3):526-36.

24. Lioumis P, Zhdanov A, Makela N, Lehtinen H, Wilenius J, Neuvonen T, et al. A novel approach for documenting naming errors induced by navigated transcranial magnetic stimulation. J Neurosci Methods. 2012;204(2):349-54.

25. Rogic $M$, Deletis $V$, Fernandez-Conejero I. Inducing transient language disruptions by mapping of Broca's area with modified patterned repetitive transcranial magnetic stimulation protocol. J Neurosurg. 2014;120(5):1033-41.

26. Sollmann N, Hauck T, Obermuller T, Hapfelmeier A, Meyer B, Ringel F, et al. Inter- and intraobserver variability in motor mapping of the hotspot for the abductor pollicis brevis muscle. BMC Neurosci. 2013;14:94.

27. Petrovich Brennan NM, Whalen S, de Morales BD, O'Shea JP, Norton IH, Golby AJ. Object naming is a more sensitive measure of speech localization than number counting: converging evidence from direct cortical stimulation and fMRI. Neuroimage. 2007;37 Suppl 1:S100-8.

28. Sollmann N, Tanigawa N, Ringel F, Zimmer C, Meyer B, Krieg SM. Language and its right-hemispheric distribution in healthy brains: an investigation by repetitive transcranial magnetic stimulation. Neuroimage. 2014;102 Pt 2:776-88.

29. Krieg SM, Tarapore PE, Picht T, Tanigawa N, Houde J, Sollmann N, et al. Optimal timing of pulse onset for language mapping with navigated repetitive transcranial magnetic stimulation. Neuroimage. 2014;100:219-36.

30. Krieg SM, Sollmann N, Hauck T, Ille S, Foerschler A, Meyer B, et al. Functional language shift to the right hemisphere in patients with language-eloquent brain tumors. PLoS One. 2013;8(9):e75403.

31. Hernandez-Pavon JC, Makela N, Lehtinen H, Lioumis P, Makela JP. Effects of navigated TMS on object and action naming. Front Hum Neurosci. 2014:8:660.

32. Wassermann EM, Blaxton TA, Hoffman EA, Berry CD, Oletsky H, PascualLeone A, et al. Repetitive transcranial magnetic stimulation of the dominant hemisphere can disrupt visual naming in temporal lobe epilepsy patients. Neuropsychologia. 1999;37(5):537-44.

33. Sparing R, Mottaghy FM, Hungs M, Brugmann M, Foltys $H$, Huber W, et al Repetitive transcranial magnetic stimulation effects on language function depend on the stimulation parameters. J Clin Neurophysiol. 2001;18(4):326-30.

34. Knops A, Nuerk HC, Sparing R, Foltys H, Willmes K. On the functional role of human parietal cortex in number processing: how gender mediates the impact of a 'virtual lesion' induced by rTMS. Neuropsychologia. 2006:44(12):2270-83.

35. Candidi M, Urgesi C, lonta S, Aglioti SM. Virtual lesion of ventral premotor cortex impairs visual perception of biomechanically possible but not impossible actions. Soc Neurosci. 2008;3(3-4):388-400.

36. Orosz A, Jann K, Wirth M, Wiest R, Dierks T, Federspiel A. Theta burst TMS increases cerebral blood flow in the primary motor cortex during motor performance as assessed by arterial spin labeling (ASL). Neuroimage. 2012;61(3):599-605.

37. Corina DP, Loudermilk BC, Detwiler L, Martin RF, Brinkley JF, Ojemann G. Analysis of naming errors during cortical stimulation mapping: implications for models of language representation. Brain Lang. 2010;115(2):101-12.

38. Mills KR, Murray NM, Hess CW. Magnetic and electrical transcranial brain stimulation: physiological mechanisms and clinical applications. Neurosurgery. 1987;20(1):164-8.

39. Pascual-Leone A, Gates JR, Dhuna A. Induction of speech arrest and counting errors with rapid-rate transcranial magnetic stimulation. Neurology. 1991:41(5):697-702.

40. Jennum P, Friberg L, Fuglsang-Frederiksen A, Dam M. Speech localization using repetitive transcranial magnetic stimulation. Neurology. 1994;44(2):269-73.

41. Lesser RP, Luders H, Klem G, Dinner DS, Morris HH, Hahn JF, et al. Extraoperative cortical functional localization in patients with epilepsy. J Clin Neurophysiol. 1987;4(1):27-53. 
42. Michelucci R, Valzania F, Passarelli D, Santangelo M, Rizzi R, Buzzi AM, et al. Rapid-rate transcranial magnetic stimulation and hemispheric language dominance: usefulness and safety in epilepsy. Neurology. 1994;44(9):1697-700.

43. Epstein CM, Meador KJ, Loring DW, Wright RJ, Weissman JD, Sheppard S, et al. Localization and characterization of speech arrest during transcranial magnetic stimulation. Clin Neurophysiol. 1999;1 10(6):1073-9.

44. DeLeon J, Gottesman RF, Kleinman JT, Newhart M, Davis C, Heidler-Gary J, et al. Neural regions essential for distinct cognitive processes underlying picture naming. Brain. 2007;130(Pt 5):1408-22.

45. Vigneau M, Beaucousin V, Herve PY, Duffau H, Crivello F, Houde O, et al. Meta-analyzing left hemisphere language areas: phonology, semantics, and sentence processing. Neuroimage. 2006;30(4):1414-32.

46. Pascual-Leone A, Houser CM, Reese K, Shotland LI, Grafman J, Sato S, et al. Safety of rapid-rate transcranial magnetic stimulation in normal volunteers. Electroencephalogr Clin Neurophysiol. 1993;89(2):120-30.

47. Rossi S, Hallett M, Rossini PM, Pascual-Leone A. Safety of TMSCG. Safety, ethical considerations, and application guidelines for the use of transcranial magnetic stimulation in clinical practice and research. Clin Neurophysiol. 2009:120(12):2008-39.

48. Wassermann EM. Risk and safety of repetitive transcranial magnetic stimulation: report and suggested guidelines from the International Workshop on the Safety of Repetitive Transcranial Magnetic Stimulation, June 5-7, 1996. Electroencephalogr Clin Neurophysiol. 1998;108(1):1-16.

49. Fauth C, Meyer BU, Prosiegel M, Zihl J, Conrad B. Seizure induction and magnetic brain stimulation after stroke. Lancet. 1992;339(8789):362.

50. Kandler R. Safety of transcranial magnetic stimulation. Lancet. 1990;335(8687):469-70.

\section{Submit your next manuscript to BioMed Central and take full advantage of:}

- Convenient online submission

- Thorough peer review

- No space constraints or color figure charges

- Immediate publication on acceptance

- Inclusion in PubMed, CAS, Scopus and Google Scholar

- Research which is freely available for redistribution 